\title{
Original
}

\section{Riesgo cardiovascular en la población con sobrepeso u obesidad en el cantón Central de Cartago, CARMEN 2001.}

\section{(Cardiovascular risk of an overweight and obese population in Central County of Cartago, CARMEN 2001)}

Marlene Roselló-Araya', Sonia Guzmán-Padilla'

\section{Resumen}

Objetivo general: Determinar el nivel de riesgo cardiovascular global de la población con sobrepeso u obesidad del cantón Central de Cartago, CARMEN 2001.

Metodología: Estudio de tipo descriptivo, transversal, basado en los datos del proyecto CARMEN, realizado en 2001 en Cartago, utilizando la tabla de Framingham clásica. Muestra 373 personas (142 hombres y 231 mujeres) con sobrepeso u obesidad, con edades comprendidas entre 30 y 64 años.

Resultados: El 61,9\% eran mujeres; la edad promedio fue de 45,1 \pm 9,4 años; el 38,8\% de la población se ubicó en el grupo de edad de 35 a 44 años. El 39,8\% de los hombres y el 18,3\% de las mujeres con sobrepeso se ubicó en riesgo cardiovascular de moderado a alto. El $73 \%$ de los hombres y el $28,2 \%$ de las mujeres con obesidad se ubicó en la categoría de riesgo cardiovascular moderado y alto. El $80,0 \%$ de los diabéticos, $21,3 \%$ de los fumadores, el $55,4 \%$ de los hipertensos, el $24,3 \%$ con colesterol elevado, el $42,9 \%$ con triglicéridos de más de $150 \mathrm{mg} / \mathrm{dl}$, el $37,2 \%$ con el LDL mayor de $130 \mathrm{mg} / \mathrm{dl}$ y el 46,6\% con HDL bajo se ubicaron en un riesgo cardiovascular de moderado a alto.

Conclusiones: Este estudio evidenció los altos porcentajes de lípidos elevados, hipertensión y fumado en esta población, lo que confirma que cuanto mayor es el grado de sobrepeso u obesidad,

Nutricionistas-Investigadoras. Instituto Costarricense de Investigación y Enseñanza en Nutrición y Salud (INCIENSA), Costa Rica

Abreviaturas: CARMEN, Conjunto de Acciones para la Reducción Multifactorial de las Enfermedades no Transmisibles; ENN, Encuesta Nacional de Nutrición; FR, factor de riesgo; HDL, high density lipoprotein; IMC, índice de masa corporal, IMC: índice de masa corporal; LDL, low density lipoprotein; OMS, Organización Mundial de la Salud; RCV, Riesgo Cardiovascular.

Correspondencia: Marlene Roselló Araya, INCIENSA,Apdo. 4-2250, Tres Ríos, Cartago, Costa Rica.mrosello@inciensa. sa.cr mayores serán también los valores de la presión sanguínea, glicemias, triglicéridos y colesterol total. El control del peso corporal es, por lo tanto, una medida clave para prevenir el riesgo de un problema cardiovascular.

Descriptores: Framingham, nivel de riesgo cardiovascular, factores de riesgo, IMC, sobrepeso, obesidad.

\section{Abstract}

Aim: To determine the global cardiovascular risk of a population with overweight and obesity from Central Cartago County, CARMEN 2001.

Methodology: Descriptive crossed study based on the data of the CARMEN Project, carried out in Cartago, in 2001, using as a reference the classic Framingham Table. Sample, 373 persons with overweight and obesity were studied there were 142 men and 231 women, aged from 30 to 64 years old showing. 
ISSN 0001-6002/2008/50/2/97-101 Acta Médica Costarricense, (C)2008 Colegio de Médicos y Cirujanos
Results: Of the total sample analyzed, $61.9 \%$ were women. The average age for the entire group was $45.1 \pm 9.4$ years. Thirty eight point $8 \%$ of the population was between 35 and 44 years. Thirty nine point $8 \%$ of the women and $18,3 \%$ of men with overweight and $73 \%$ of obese men and $28.2 \%$ of obese women were included in the group with a moderate or high cardiovascular risk. The group with moderate to high cardiovascular risk also included $80,21.3$ and $55.4 \%$ of the patients showing diabetes, smoking habits and hypertension, respectively. The same risk was described for the patients having high serum cholesterol (24.3\%), serum triglycerides over 150 $\mathrm{mg} / \mathrm{dL}(42.9 \%)$, serum LDL over $130 \mathrm{mg} / \mathrm{dL}(37.2 \%)$ and low HDL (46.6\%).

Conclusions: This study showed a high incidence of elevated blood lipid levels, hypertension and smoking habits in this population. These findings also confirmed that the higher the overweight and obesity as observed in this group, the higher the risk of hypertension, diabetes and elevated serum triglycerides and cholesterol levels. Consequently, body weight control appears as a key factor to diminish the cardiovascular risk in the population.

Keywords: Framingham, cardiovascular risk, risk factors, body mass index, overweight, obesity, LDL, HDL

\section{Recibido: 1 de diciembre de 2006 Aceptado: 23 octubre de 2007}

La obesidad, según la Organización Mundial de la Salud (OMS), se ha convertido en una auténtica pandemia, constituyéndose en uno de los principales retos actuales para la salud pública y el más frecuente de los problemas nutricionales. ${ }^{1}$

Existen evidencias de que la obesidad de grado ligero da lugar al aumento de la mortalidad cardiovascular. En los hombres el $10 \%$ de exceso del peso corporal provoca el un aumento del $30 \%$ en el riesgo coronario, debido especialmente al efecto de la obesidad sobre otros factores de riesgo (FR).

El sobrepeso y la obesidad no son considerados como enfermedades por sí solas. En Costa Rica la situación es muy similar a la que muestran las estadísticas mundiales y preocupa el aumento que se ha producido a lo largo de los años. Varios estudios, como son las encuestas nacionales de nutrición $(\mathrm{ENN})^{2}$ realizadas desde 1966, han evaluado los FR. A partir de estas se reportó que el $45 \%$ de las mujeres de 20 a 44 años y el $75 \%$ de los mayores de 45 años presentaron sobrepeso u obesidad. En la encuesta de $1982,{ }^{3}$ un $34,6 \%$ de la población presentó sobrepeso y obesidad, el cual aumentó al 45,9\% en 1996, en las mujeres de 20 a 44 años. ${ }^{4}$ Para el grupo de 45 a 59 años la prevalencia aumentó del 55,6\% al $75 \%$, de 1982 a $1995 .^{5}$

En la encuesta de 1999, en el distrito de Damas en Desamparados, y en 2000, en San Antonio de Nicoya, se determinó que un $56,4 \%$ de las mujeres y un $58,8 \%$ de los hombres de Damas con edades entre 19 y 59 años, presentaron algún grado de sobrepeso u obesidad, mientras que en Nicoya fueron el $58,8 \%$ y el $40,6 \%$, respectivamente. ${ }^{6}$

Con la finalidad de mejorar la salud de las poblaciones en las Américas, la Organización Panamericana de Salud (OPS) creó la Red Panamericana, conformada por instituciones gubernamentales, no gubernamentales, académicas y del sector privado, llamada CARMEN
(Conjunto de Acciones para la Reducción Multifactorial de las Enfermedades no transmisibles), a la que se unió Costa Rica a partir de 1999. ${ }^{7}$ En 2001, CARMEN realizó en nuestro país la encuesta basal de factores de riesgo para enfermedades no transmisibles, en el área urbana del cantón central de Cartago, estudio que ratificó los altos índices de sobrepeso (20,6\% de las personas de 15 a 19 años y $59,4 \%$ mayores de 20 años) y de obesidad, los cuales aumentaron con la edad, así como la presencia y aumento del resto de factores de riesgo en esta población. ${ }^{8}$

Para valorar la magnitud de la interacción entre los distintos FR, Estados Unidos y Europa han aplicado a sus poblaciones el cálculo de riesgo cardiovascular (RCV), método matemático que estima la probabilidad que tiene un individuo de presentar una enfermedad cardiovascular en un período determinado, generalmente 10 años. Este método se basa en la cohorte del estudio de Framingham. ${ }^{9,10}$

El objetivo del estudio fue determinar el riesgo cardiovascular global de la población con sobrepeso u obesidad del cantón Central de Cartago.

\section{Metodología}

Se realizó un estudio de tipo descriptivo, transversal, utilizando la base de datos de la encuesta basal del proyecto CARMEN, efectuada en Cartago en 2001, la cual fue suministrada por los encargados de esta, y con aprobación del Comité Ético de INCIENSA. Se seleccionaron las personas de 30 a 64 años, con sobrepeso (índice de masa corporal; IMC) de 25 a 29,9$) \mathrm{kg} / \mathrm{m}^{2}$ y obesidad (IMC $\geq 30$ ) $\mathrm{kg} / \mathrm{m}^{2}$. Se excluyeron aquellas que presentaron un IMC menor o igual a 24,9 , para una muestra final de 373 personas (142 hombres y 231 mujeres). 
Las variables consideradas para este estudio fueron: sexo, edad en años, educación, ocupación, tabaquismo, Diabetes mellitus, hipertensión arterial, colesterol total, LDL-colesterol y HDL-colesterol, así como las variables antropométricas de peso y talla, con el cual se calculó el IMC: peso corporal $\left(\mathrm{Kg}\right.$.) / talla $\left(\mathrm{m}^{2}\right)$.

En el análisis del riesgo cardiovascular se utilizó el método de puntuación (para cada variable) y las categorías de riesgo establecidas en la tabla de Framingham clásica, donde riesgo alto es $\geq 20 \%$, riesgo moderado es $10-19 \%$ y riesgo bajo, $\leq 9 \%{ }^{9}$

Los datos obtenidos se almacenaron en el programa Microsoft Excel $2002^{\circledR}$ y se analizaron con el programa SPSS $^{\circledR}$, versión $10,0, y$ se expresan con estadística descriptiva.

\section{Resultados}

El 64,3\% del total de la muestra presentó sobrepeso y el $35,7 \%$, obesidad. La edad promedio fue de 45,1 \pm 9,4 años; el $61,9 \%$ eran mujeres. Un 38,8\% se ubicó en el grupo de edad de 35 a 44 años. En lo que respecta a ocupación, el $36,3 \%$ se dedicaba a los oficios del hogar el 48,0\% eran trabajadores renumerados y 48,3\% contaban con estudios primarios.
El peso, la talla y el IMC medio de la población fue de $75,0 \pm 11,7 \mathrm{Kg}, 159,6 \pm 8,7 \mathrm{~cm}$ y de $29,4 \pm 3,7 \mathrm{Kg} / \mathrm{m}^{2}$ respectivamente. Los hombres presentaron un peso promedio de $80,6 \pm 9,9$, con una talla de $167,6 \pm 6,5 \mathrm{~cm}$ y un IMC de $28,6 \pm 2,6$, y las mujeres, de 71,5 $\pm 11,4 \mathrm{Kg}$ de peso, con una estatura de $154,7 \pm 5,6 \mathrm{~cm}$ y un IMC de $29,9 \pm 4,2 \mathrm{Kg} / \mathrm{m}^{2}$.

En el Cuadro 1 se muestran los principales factores de riesgo presentes en esta población. Se observan diferencias estadísticamente significativas por sexo, en la presión arterial sistólica y diastólica, así como en las HDL, triglicéridos y el IMC.

El $67,3 \%$ de la población presentó RCV bajo (50,0\% hombres y 77,9\% mujeres). El 39,8\% de los hombres con sobrepeso se ubicó en RCV de moderado a alto, siendo este porcentaje menor en las mujeres con sobrepeso (18,3\%). En el caso de la población obesa, un $73 \%$ de los hombres y un $28,2 \%$ de las mujeres se ubicó en la categoría de RCV moderado y alto (Cuadro 2).

En las figuras 1 y 2, se observa la distribución de la población según IMC por sexo y grupo de edad. Más del $60 \%$ de los hombres y el $67,6 \%$ de las mujeres con sobrepeso u obesidad eran menores de 54 años de edad. Al analizar los grupos de edad por nivel de RCV, se determinó que más del $65 \%$ de las personas, tanto hombres como mujeres menores de 44 años, se ubicaron en el grupo de RCV bajo, mientras que los mayores de 45 años eran de RCV moderado (Figuras 3 y 4$)$.

\begin{tabular}{|c|c|c|c|c|c|}
\hline \multicolumn{6}{|c|}{$\begin{array}{l}\text { Cuadro 1. Características de la población estudiada, según factores de riesgo } \\
\text { cardiovascular, en la población CARMEN, } 2001\end{array}$} \\
\hline \multirow{2}{*}{\multicolumn{2}{|c|}{ Factores de riesgo }} & Total & Sobrepeso & Obesidad & $p$ \\
\hline & & $n=373$ & $n=240$ & $n=133$ & \\
\hline \multicolumn{2}{|c|}{ Edad (x \pm d.e.) } & $45,1 \pm 9,7$ & $44,7 \pm 9,3$ & $45,7 \pm 9,8$ & 0,33 \\
\hline \multirow[t]{2}{*}{ Sexo } & Hombres & 142 & $98(69,0 \%)$ & $44(31,0 \%)$ & - \\
\hline & Mujeres & 231 & $142(61,5 \%)$ & $89(38,5 \%)$ & - \\
\hline \multicolumn{2}{|c|}{ Diabetes mellitus ( $\mathrm{n}$ ) } & 15 & $5(33,3 \%)$ & $10(66,7 \%)$ & - \\
\hline \multicolumn{2}{|c|}{ Tabaquismo } & 99 & $63(63,6 \%)$ & $36(36,4 \%)$ & - \\
\hline \multicolumn{2}{|c|}{ Hipertensión } & 103 & $53(51,5 \%)$ & $50(48,5 \%)$ & - \\
\hline \multicolumn{2}{|c|}{ Presión arterial sistólica (mmHg) } & $123,0 \pm 21,1$ & $120,6 \pm 19,7$ & $127,5 \pm 22,8$ & 0,002 \\
\hline \multicolumn{2}{|c|}{ Presión arterial diastólica $(\mathrm{mmHg})$} & $78,8 \pm 11,0$ & $77,4 \pm 10,5$ & $81,4 \pm 11,4$ & 0,001 \\
\hline \multicolumn{2}{|c|}{ Colesterol total (mg/dl) } & $226,8 \pm 40,8$ & $227,7 \pm 39,9$ & $225,0 \pm 41,7$ & 0,53 \\
\hline \multicolumn{2}{|c|}{ HDL-colesterol (mg/dl) } & $40,9 \pm 13,5$ & $42,1 \pm 14,4$ & $38,7 \pm 11,5$ & 0,019 \\
\hline \multicolumn{2}{|c|}{ LDL-colesterol (mg/dl) } & $145,5 \pm 36,1$ & $146,9 \pm 37,7$ & $142,9 \pm 32,9$ & 0,303 \\
\hline \multicolumn{2}{|c|}{ Triglicéridos (mg/dl) } & $201,7 \pm 110,4$ & $193,5 \pm 99,3$ & $217,0 \pm 129,9$ & 0,04 \\
\hline \multicolumn{2}{|l|}{ IMC } & $29,4 \pm 3,7$ & $27,2 \pm 1,4$ & $33,3 \pm 3,4$ & 0,000 \\
\hline
\end{tabular}

Datos expresados como media y desviación estándar (entre paréntesis) para las variables cuantitativas y como número y porcentaje (entre paréntesis) para variables cualitativas 


\begin{tabular}{|c|c|c|c|c|c|c|c|c|c|c|c|c|c|c|}
\hline \multicolumn{15}{|c|}{$\begin{array}{l}\text { Cuadro 2. Clasificación del estado nutricional de la población, } \\
\text { según nivel de riesgo cardiovascular, por sexo }\end{array}$} \\
\hline & \multicolumn{4}{|c|}{ Sobrepeso } & \multicolumn{4}{|c|}{ Obesidad } & \multicolumn{4}{|c|}{ Total } & \multicolumn{2}{|c|}{ Total } \\
\hline & \multicolumn{2}{|c|}{ Hombres } & \multicolumn{2}{|c|}{ Mujeres } & \multicolumn{2}{|c|}{ Hombres } & \multicolumn{2}{|c|}{ Mujeres } & \multicolumn{2}{|c|}{ Hombres } & \multicolumn{2}{|c|}{ Mujeres } & & \\
\hline & $\mathrm{n}$ & $\%$ & $\mathrm{n}$ & $\%$ & $\mathrm{n}$ & $\%$ & $\mathrm{n}$ & $\%$ & $n$ & $\%$ & $n$ & $\%$ & $n$ & $\%$ \\
\hline Bajo & 59 & 60,2 & 116 & 81,7 & 12 & 27,0 & 64 & 72 & 71 & 50,0 & 180 & 77,9 & 251 & 67,3 \\
\hline Moderado & 27 & 27,6 & 23 & 16,2 & 21 & 48,0 & 23 & 26 & 48 & 33,8 & 46 & 19,9 & 94 & 25,2 \\
\hline Alto & 12 & 12,2 & 3 & 2,1 & 11 & 25,0 & 2 & 2,2 & 23 & 16,2 & 5 & 2,2 & 28 & 7,5 \\
\hline Total & 98 & 100 & 142 & 100 & 44 & 100 & 89 & 100 & 142 & 100 & 231 & 100 & 373 & 100 \\
\hline
\end{tabular}

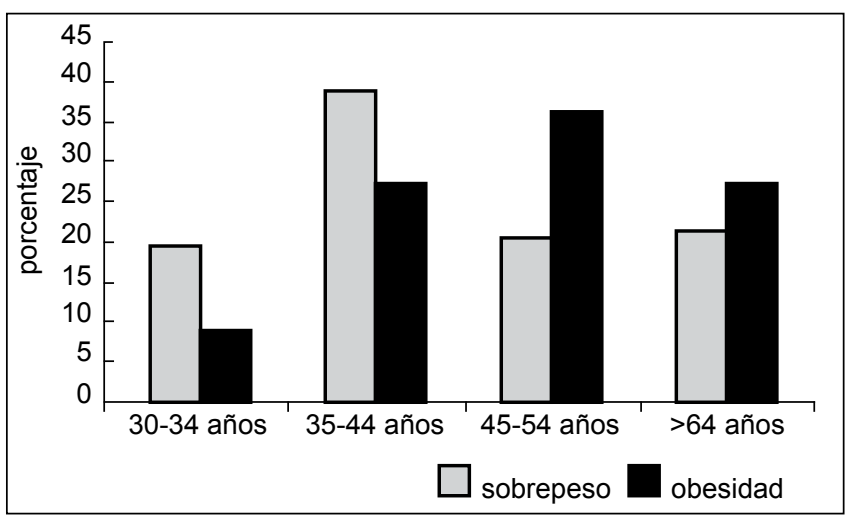

Figura 1. Clasificación de los hombres con sobrepeso y obesidad, según grupo de edad.

En la figura 5 se presentan los factores de riesgo y su clasificación, según nivel de RCV. Se encontró que el 80,0\% de los diabéticos, el $21,3 \%$ de los fumadores, el $55,4 \%$ de los hipertensos, el $24,3 \%$ con colesterol elevado, el $43 \%$ con triglicéridos demás de $150 \mathrm{mg} / \mathrm{dl}$, el 37,2\% con LDL elevado y el $46,6 \%$ con HDL bajo se ubicaron en un RCV de moderado a alto. Por sexo, tanto la mayoría de hombres como de mujeres se ubicó con estas mismas condiciones descritas en bajo RCV. Según factor de riesgo, se evidencia que el de mayor peso en la clasificación de riesgo moderado es la presencia de diabetes y de hipertensión.

\section{Discusión}

Está comprobada la una relación entre el sobrepeso y la morbimortalidad de origen cardiovascular tanto en hombres como en mujeres. En la actualidad, el sobrepeso y la obesidad son las enfermedades nutricionales más frecuentes y, además, factores independientes secundarios modificables, asociados habitualmente a otros factores de riesgo. ${ }^{10}$

En nuestro estudio el IMC promedio fue de $28,6 \mathrm{Kg} . / \mathrm{m}^{2}$ en hombres y de $29,9 \mathrm{Kg} . / \mathrm{m}^{2}$ en mujeres, por lo que esta población podría estar expuesta a incrementar su riesgo coronario. Un estudio realizado en Finlandia en 1996

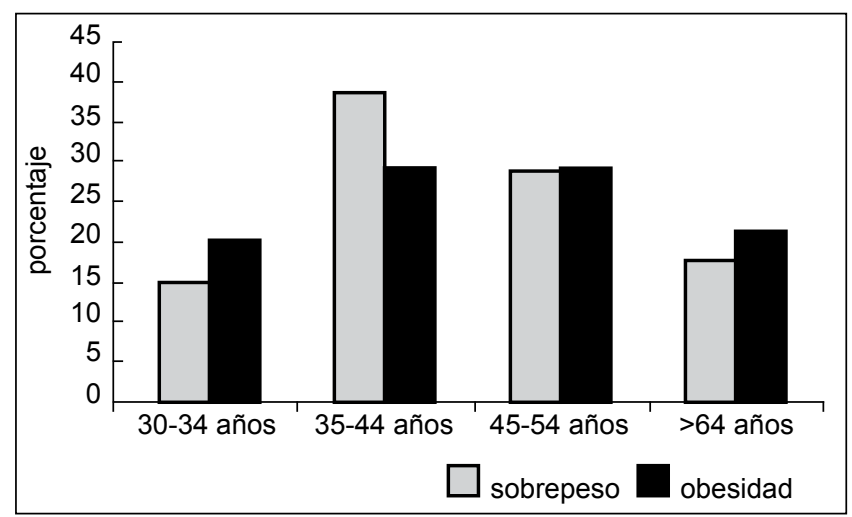

Figura 2. Clasificación de las mujeres con sobrepeso y obesidad, por grupo de edad

demostró que la mortalidad coronaria se duplicaba conforme aumentaba el IMC de las personas. ${ }^{11}$

Una tercera parte de la población aquí estudiada tiene obesidad $(35,7 \%)$ y se presenta en los grupos de mayor edad y en el sexo masculino, situación que debe preocupar, ya que se conoce que los incrementos de peso durante la edad adulta son los que producen mayores efectos adversos. Se ha demostrado que los hombres con un $20 \%$ de exceso del peso deseable muestran un incremento del $20 \%$ en la probabilidad de muerte por todas las causas, un $25 \%$ en la mortalidad por enfermedad coronaria y $10 \%$ por accidentes cerebrovasculares. ${ }^{11}$

Es importante resaltar que hay estudios que indican que las personas en condiciones de sobrepeso $\mathrm{u}$ obesidad tienen un mayor riesgo de padecer enfermedad coronaria, y recomiendan que quienes la padecen inicien cuanto lo antes un tratamiento para combatirla. Esta se asocia con la aparición de otras enfermedades, tales como la hipertensión arterial o la aterosclerosis, que pueden desencadenar un evento cardíaco grave. Se estima que un $40 \%$ de la incidencia de tales afecciones se debe al sobrepeso. ${ }^{12}$

En lo que respecta a factores de riesgo, el estudio en esta población evidenció los altos porcentajes de lípidos elevados, hipertensión y fumado. Datos del estudio Framingham acerca del corazón, indican que al aumentar de peso, crece la 


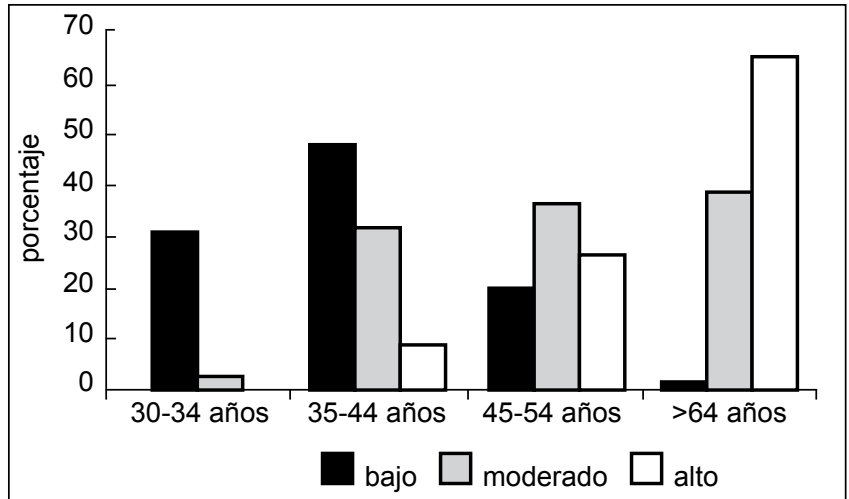

Figura 3. Clasificación de la población masculina con sobrepeso y obesidad, según nivel de riesgo y grupo de edad.

tendencia a que se agrupe un conjunto de factores predisponentes del desarrollo de enfermedades cardiovasculares, como presión arterial elevada, colesterol alto e intolerancia a la glucosa, y concluye que cuanto mayor es el grado de sobrepeso, mayores serán tambienlos valores de la presión sanguínea, la resistencia a la insulina, el nivel de triglicéridos y el colesterol total. El control del peso corporal es, por lo tanto, una medida clave para prevenir el riesgo de un problema cardiovascular. ${ }^{13}$

\section{Referencias}

1. López C, Martínez M, Sánchez A, Martínez J. 2006. Influencia de la pérdida de peso y la actividad física sobre factores de riesgo cardiovascular de mujeres obesas. Respyn [Revista digital electrónica] 2006 [citado 5 junio 2006] Disponible URL: http://www.respyn.uanl. $\mathrm{mx} / \mathrm{vii} / 1 /$ articulos/influencia.htm

2. Díaz C, Brenes H, Córdoba M, García P, Quirós J. Encuesta nutricional antropométrica y de hábitos alimentarios en Costa Rica. San José, Costa Rica: Ministerio de Salud, 1978.

3. Ministerio de Salud. Encuesta nacional de nutrición. San José, Costa Rica: Ministerio de Salud, 1982.

4. Castro G, Meza N, Monge R, Rodríguez N, Rojas K. I Encuesta nacional sobre consumo aparente de alimentos. San José, Costa Rica: Ministerio de Salud, 1989.

5. Rodríguez N, Meza N. Fascículo de consumo aparente. II encuesta nacional sobre consumo aparente de alimentos. Costa Rica, Ministerio de Salud, 1996.

6. Ministerio de Salud, INCIENSA. Encuesta basal comunidades centinela en alimentación y nutrición 1999-2000. San José, Costa Rica: Ministerio de Salud, 2003.

7. Organización Panamericana de la Salud (OPS). Red Panamericana Conjunto de Acciones para la Reducción Multifactorial de las Enfermedades no transmisibles (CARMEN) [Monografía en internet] [citado 12 de abril 2006] Disponible: http://www.paho.org/Spanish/ $\mathrm{AD} / \mathrm{DPC} / \mathrm{NC} /$ carmen-info.htm.

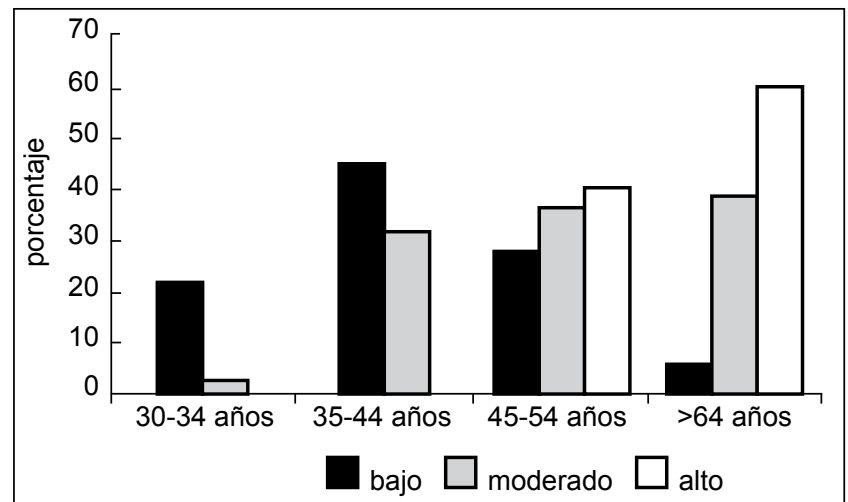

Figura 4. Clasificación de la población femenina con sobrepeso y obesidad, según nivel de riesgo y grupo de edad.

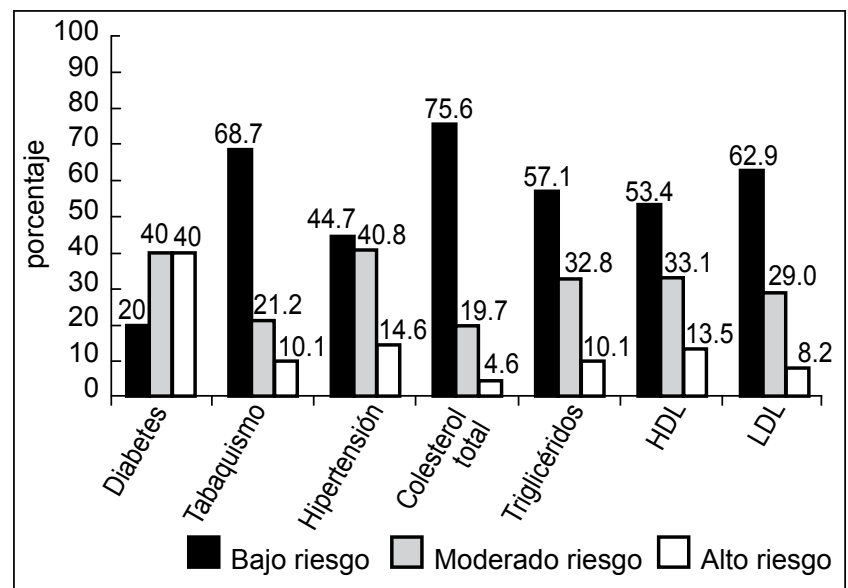

Figura 5. Factores de riesgo cardiovascular en población total con sobrepeso u obesidad, según nivel de riesgo cardiovascular.

8. Ministerio de Salud. Encuesta basal de factores de riesgo para enfermedades no transmisibles en la ciudad de Cartago, 2001. Factores alimentarios nutricionales, Costa Rica. San José, Costa Rica: Ministerio de Salud, 2003.

9. National Cholesterol Education Program. Executive Summary of the report of the National Cholesterol Education Program (NCEP) Expert Panel on Detection, Evaluation, and Treatment of High Blood Cholesterol in Adult (Adult Treatment Panel III). JAMA 2001;285:1883-1889.

10. Robson J, Boomla K, Hart B, Feder G. Estimating cardiovascular risk for primary prevention: outstanding questions for primary care. BMJ 2000;320:702-704.

11. Jousilahti P, Tuomilehto J, Vartiainen E, Pekkanen J, Puska P. Body weight, cardiovascular risk factors, and coronary mortality. 15-year follow-up of middle-age men and women in Eastern Finland. Circulation 1996;93:1372-1379.

12. Nowoszynski Norma. Advierten que la obesidad aumenta el riesgo cardiovascular. [citado 16 agosto 2006] Disponible en: http://www. turemanso.com.ar/aire/ciencia/corazon.html 